\title{
Médecine de l'addiction: naissance d'une nouvelle discipline
}

Jacques Besson, Philip Bruggmann, Thomas Bischoff, Barbara Broers, Jean-Bernard Daeppen, Jean-Alain Dubois, Jean-Pierre Gervasoni, Robert Hämmig, Martine Monnat

Groupe interdisciplinaire concerné par la médecine de l'addiction

\begin{abstract}
Aujourd'hui seul un patient sur dix touché par une problématique d'addiction est traité. La Suisse doit se doter d'un système promouvant une médecine de l'addiction permettant d'identifier et de traiter la grande diversité des troubles rencontrés dans les lieux de soins les plus touchés. Les auteurs soutiennent la création d'une médecine de l'addiction à large spectre.
\end{abstract}

\section{Une pandémie}

Les chiffres publiés par l'Organisation Mondiale de la Santé en 2000 indiquent que le tabac, l'alcool et les drogues illicites contribuent dans le monde à $12,4 \%$ de la mortalité et à $8,9 \%$ du nombre d'année de vie

Résumé

Les addictions ont fortement consolidé leur place dans le champ de la médecine à la fin du $20^{\mathrm{e}}$ siècle parce que la certitude de leurs fondements neurobiologiques était désormais acquise et que les preuves de l'efficacité des stratégies de prévention et de traitement étaient suffisantes. La médecine de l'addiction couvre un champ très large de l'activité médicale allant de la prévention de l'usage à risque, en passant par le dépistage des formes peu sévères de dépendance au traitement des formes les plus graves, chroniques et invalidantes. Cette activité médicale prend place dans une grande diversité de lieux de soins, en médecine de premier recours, particulièrement dans les cabinets de médecine de famille, mais aussi aux urgences et en pédiatrie. La médecine de l'addiction est très présente en psychiatrie où ses formes sont souvent sévères. Malgré cela, aujourd'hui seul un patient sur dix touché par une problématique d'addiction est traité. C'est la raison pour laquelle la Suisse, comme c'est le cas aux Etats-Unis et aux Pays-Bas par exemple, doit se doter d'un système promouvant une médecine de l'addiction à "large spectre», permettant d'identifier et de traiter la grande diversité des troubles rencontrés dans les lieux de soins les plus touchés. En Suisse, les médecins sont actuellement insuffisamment préparés pour assumer ce rôle. C'est aux disciplines concernées de proposer des filières de formation. Cette stratégie contribuera à améliorer les compétences de l'ensemble du corps médical dans ce domaine, avec pour conséquence d'offrir au plus grand nombre de patients touchés par les addictions les traitements les plus efficaces. perdues en raison de décès ou d'invalidité. Ces chiffres sont beaucoup plus élevés en Europe, par exemple, pour les hommes, 33,4\% des nombre d'années de vie perdues en raison de décès ou d'invalidité sont attribuables aux effets du tabac, de l'alcool et des drogues illégales (www.who.int/substance_abuse/ facts/global_burden).

\section{Touchant un pan très vaste de la médecine}

Au cours des dernières décennies, un grand nombre de données scientifiques démontrant l'efficacité des traitements des addictions ont été publiées. Les progrès scientifiques font des addictions des maladies neurologiques dont les complications touchent un grand nombre de disciplines médicales: psychiatrie, traumatologie, médecine générale, médecine d'urgence, entre autres.

Les progrès des neurosciences de l'addiction ont pour corollaire le concept d'addiction comme maladie chronique récidivante $[1,2]$, comparable à d'autres maladies chroniques récidivantes telles que l'hypertension artérielle ou le diabète. L’intérêt de cette comparaison est de mettre les addictions au cœur de la pratique médicale. L'inconvénient est de focaliser la médecine de l'addiction sur ses formes les plus graves. Il est important d'examiner le champ de cette médecine dans toute l'étendue de ses manifestations. Par exemple, la majeure partie des coûts médico-sociaux de l'alcool sont liés à l'usage à risque plutôt qu'à la dépendance, simplement parce que l'usage à risque est beaucoup plus fréquent que la dépendance [3]. La démonstration 


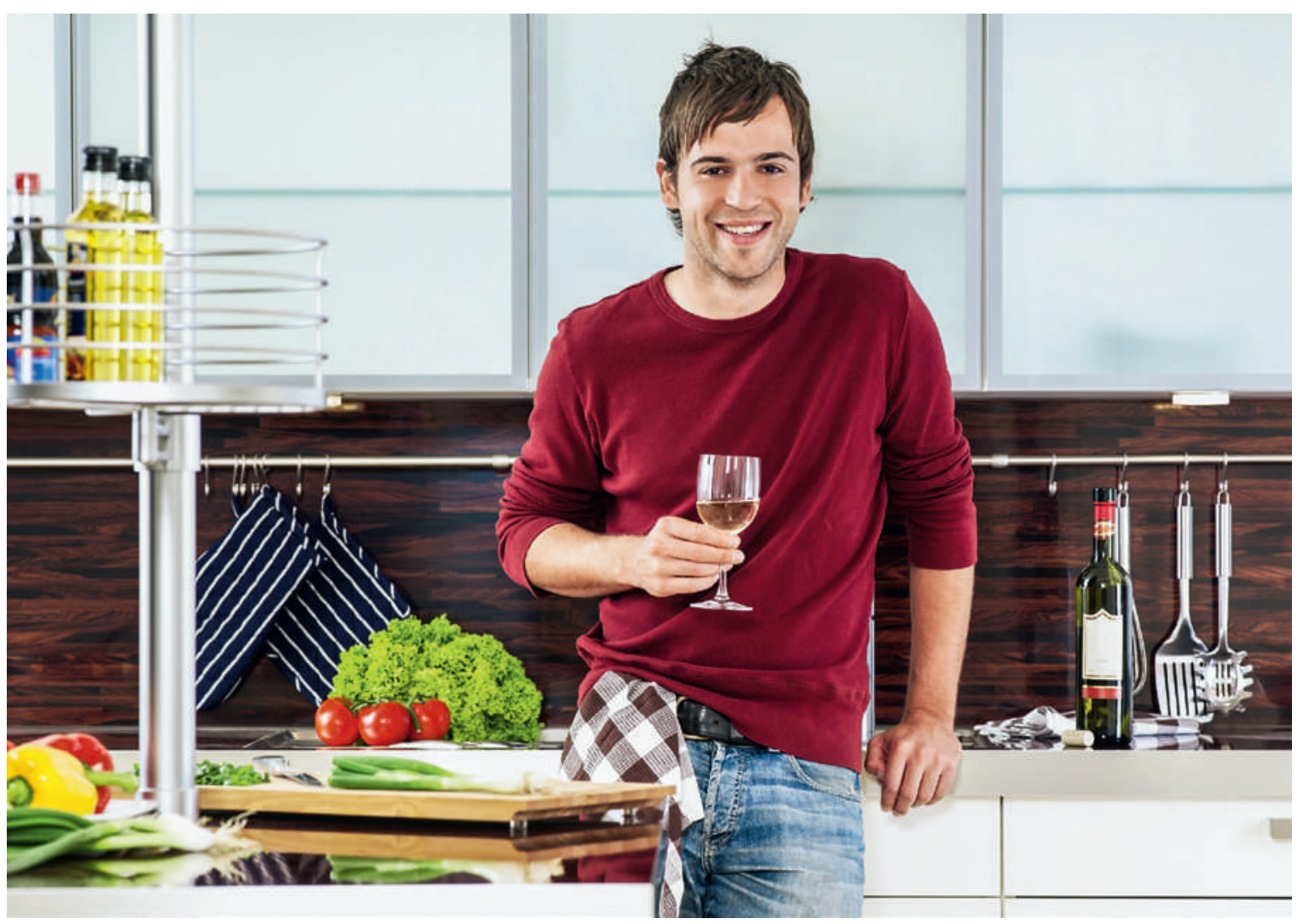

La majeure partie des coûts médico-sociaux de l'alcool sont liés à l'usage à risque plutôt qu'à la dépendance, parce que l'usage à risque est beaucoup plus fréquent que la dépendance.

de l'efficacité des stratégies de prévention ciblée sur des usages à risque ou à des dépendances de faible intensité incite à ce que la médecine de l'addiction couvre un large spectre - avant tout la médecine de famille, la psychiatrie, les urgences, la pédiatrie, mais bien sûr aussi la médecine de santé publique et la médecine préventive.

La médecine de famille a un rôle central en médecine des addictions à tout âge, que ce soit pour le dépistage et la prise en charge du tabagisme, l'alcoolisation aiguë des jeunes, les abus de somnifères en $3^{\mathrm{e}}$ âge, mais aussi des formes peu symptomatiques de dépendance aux drogues illégales. La médecine d'urgence est en première ligne des intoxications aiguës. La médecine préventive et de santé publique jouent un rôle central dans la médecine des addictions: elle en détermine l'importance en termes de prévalence et de répercussions; elle élabore et teste l'efficacité des stratégies de prévention. Les addictions occupent une place majeure en psychiatrie compte tenu de leur prévalence très élevée et des formes sévères observées chez les patients psychiatriques. La psychiatrie est au centre de la médecine des addictions, dans le sens où c'est essentiellement de son champ de recherche fondamentale, les neurosciences, et clinique, les psychothérapies, qu'émergent les traitements des addictions.

\section{Une médecine de l'addiction fondée sur les preuves}

L'amélioration de la compréhension des mécanismes neurobiologiques des addictions a contribué au développement de traitements pharmacologiques efficaces, par exemple la méthadone et la buprénorphine dans le traitement du mésusage d'opiacés, la naltrexone dans celui de la dépendance à l'alcool et la varénicicline pour la dépendance à la nicotine. L'efficacité du dépistage et de l'intervention brève pour diminuer la consommation d'alcool et d'autres drogues, en particulier en médecine de premier recours, a aussi été bien démontrée [4]. La recherche a montré également l'efficacité de différentes formes de "counselling" tels que la prévention de la rechute et l'entretien motivationnel. Une utilisation plus large des traitements fondés sur les preuves contribuera à une réduction des dépenses liées aux maladies résultant directement (par exemple le cancer du poumon) ou indirectement (par exemple l'hépatite $\mathrm{C}$ ), aux accidents, à la perte de productivité, à la criminalité, aux souffrances 
psychiques des patients et de leurs proches. Le défaut actuel du système de santé d'adresser efficacement les troubles liés aux addictions interfère avec le résultat des traitements médicaux de nombreuses maladies, par exemple la BPCO et le HIV.

\section{Le «treatment gap»}

Malgré l'apparition de ces traitements, les études américaines suggèrent qu'une faible proportion de patients présentant des addictions (aux drogues légales et illégales) sont traités, des chiffres probablement assez proches de la situation suisse. Par

\section{Le «treatment gap» est largement dû au manque de formation des soignants.}

exemple, parmi les quelques 23 millions d'Américains qui auraient besoin de traitement spécialisé pour les addictions, seulement 2,5 millions (11\%) en bénéficient [5]. Ce «treatment gap»est largement dû au manque de formation des soignants. En 2014, à la demande de l'Office fédéral de la santé publique, les Docteurs Catherine Ritter et Thomas Bischoff de l'Institut Universitaire de Médecine Générale de Lausanne ont recensé l'enseignement prégradué de la médecine de l'addiction dans les facultés de médecine suisses. Leur rapport conclut à la recommandation, comme ce fut le cas en 2008 aux USA, de développer en Suisse un enseignement de la médecine de l'addiction ayant la même priorité que les autres disciplines médicales et des méthodes d'enseignement comparables.

\section{Une nouvelle discipline médicale}

Aux Etats-Unis, la création en 1970 d'un institut de recherche sur le traitement des drogues et de l'alcool (National Institute on Drug Abuse et National Institute on Alcool Abuse and Alcoholism) a été suivi par une explosion des connaissances dans le domaine. Cela a conduit en 1991 à la création d'une sous-spécialité de la psychiatrie de l'addiction. En 2007, la médecine de l'addiction est devenue une spécialité indépendante (American Board of Addiction Medicine). Elle propose une formation multidisciplinaire qui inclut la médecine d'urgence, la médecine de famille, la médecine interne, l'obstétrique, la pédiatrie, la psychiatrie, la médecine préventive et la chirurgie. Son programme de formation comprend des sciences de base incluant des aspects génétiques, pharmacologiques, neurobiologiques, d'épidémiologie et de prévention avant de se focaliser sur des concepts cliniques: dépistage, intervention brève et traitement spécifique des dépendances à l'alcool, aux drogues illégales, à la nicotine, traitement résidentiel et ambulatoire, sevrage, intervention psychosociale, traitement des comorbidités médicales et psychiatriques, aspects sociaux et légaux. Une sous-spécialité en médecine de l'addiction au sein de la formation postgraduée en médecine de famille est à l'étude [5]. Les Pays-Bas on également créé une formation spécifique en médecine de l'addiction [6]. La France va adopter une formation spécialisée transversale en addictologie, formation théorique et pratique sur deux ans, qui sera accessible aux médecins spécialisés ou en voie de l'être, en gastro-entérologie, médecine interne, médecine générale, médecine du travail, pneumologie, psychiatrie, gériatrie et santé publique.

\section{Recommandations du groupe interdisciplinaire}

La Suisse doit relever le défi de la médecine de l'addiction en proposant des filières de formation permettant aux médecins confrontés quotidiennement aux addictions de proposer au plus grand nombre de patients les traitements dont l'efficacité est avérée. Les signataires de cet article soutiennent la création d'une médecine de l'addiction à large spectre incluant des programmes de formation continue qui permettent de répondre aux besoins diversifiés des groupes cibles.

\section{Références}

1 McLellan AT, Lewis DC, O'Brien CP, Kleber HD. Drug dependence, a chronic medical illness: implications for treatment, insurance, and outcomes evaluation. JAMA. 2000;284(13):1689-95.

2 McLellan AT. Have we evaluated addiction treatment correctly? Implications from a chronic care perspective. Addiction. 2002;97(3):249-52.

3 Kreitman N. Alcohol consumption and the preventive paradox. Br J Addict. 1986;81(3):353-63.

4 Bertholet N, Daeppen JB, Wietlisbach V, Fleming M, Burnand B. Reduction of alcohol consumption by brief alcohol intervention in primary care: systematic review and meta-analysis. ArchInternMed. 2005;165(9):986-95.

5 O'Connor PG, Sokol RJ, D'Onofrio G. Addiction medicine: the birth of a new discipline. JAMA internal medicine. 2014;174(11):1717-8.

6 De Jong C, Luycks L, Delicat JW. The master in addiction medicine program in the Netherlands. Substance abuse: official publication of the Association for Medical Education and Research in Substance Abuse. 2011;32(2):108-14. 